\title{
Impact of different exercise training on mitochondrial in diabetic nephropathy
}

\begin{abstract}
The purpose of this study was to compare the effects of aerobic, resistance and combined exercise training on mitochondrial dysfunction in diabetic nephropathy in rats. Male Wistar rats were divided into eight groups: non-diabetics (untrained, trained aerobic, trained resistance, trained combined), diabetic (untrained, trained aerobic, trained resistance, trained combined). Serum glucose levels, maximum effort test, renal function and mitochondrial biogenesis were assessed. Western blot and immunohistochemistry were used to evaluate the expression of the mitochondrial biogenesis protein and Acetil-CoA carboxylase. No differences were observed in body weight and glycaemia between diabetic rats. Diabetic animals and controls in the trained aerobic and combined groups exhibited an increase in running time, whereas animals in the trained resistance and combined groups showed greater strength. Our data suggest that exercise training can modulate the molecular pathways involved in mitochondrial biogenesis and control of renal system, decreasing the progression of diabetic nephropathy and indicating that renal disease can be prevented with non- pharmacological treatments. In conclusion, the three training protocols led to an increase in physical capacity and mitochondrial and renal benefits that were not dependent on improvements in glycemic control. In this model, combined training seems to promote more benefits than resistance or aerobic training alone.
\end{abstract}

Keywords: exercise training, nephropathy, diabetes, mitochondrial
Volume 8 Issue 2 - 202 I

\section{Luciana Jorge, Natalia Lopes Reinecke, Wesley Henrique Da Silva, Maria Lucia SG Jorge, Nestor Schor (in Memoria) \\ Nephrology Division, Department of Medicine, Federal University of São Paulo, Brazil}

Correspondence: Luciana Jorge, Nephrology Division, Department of Medicine, Federal University of Sao Paulo Rua Botucatu, $740,2^{\circ}$ andar, Disciplina de Nefrologia, 04023-900 São Paulo, SP, Brazil, Tel +55 II 94592 I554, Fax +55 5904684, Email luciana.jorge@unifesp.br

Received: August 25, 2021 | Published: September 29, 2021
Abbreviations: DKD, diabetic kidney disease; DN, diabetic nephropathy; Mfn2, Mitofusiun 2; Drp-1, dynamic-related protein 1; ACC, acetyl-CoA carboxylase; UN, untrained; TA, trained aerobic; $\mathrm{TR}$, trained resistance; TC, trained combined; HR, horseradish peroxidase; ECL, electrochemiluminescence

\section{Introduction}

Diabetes is closely linked to a group of pathologies such as hypertension, endothelial dysfunction, and obesity that involve macrovascular complications such as atherosclerosis, and microvascular complications such as neuropathy, retinopathy, angiopathy and diabetic kidney disease (DKD) ${ }^{1,2}$ Diabetic nephropathy (DN) is the leading cause of end-stage renal disease and can be characterized by a proapoptotic and prooxidative environment. The first alteration induced by diabetes associated with kidney dysfunction is the tubular injury. ${ }^{3,4}$ The mitochondrial quality control depends on proteins upstream the AMP- activated protein kinase (AMPK), which is present in all mammalian cells and is the principal conserved fuelsensing enzyme. ${ }^{5,6}$ While the downstream protein Mitofusiun 2 (Mfn2) controls fusion of the mitochondrial outer membrane, dynamic-related protein 1 (Drp-1) works on the mitochondrial fission process..$^{7,8,9}$ The peroxisome proliferator-activated receptor gamma coactivator 1-alpha (PGC-1 $\alpha$ ) is the principal protein involved in mitochondrial biogenesis. $^{10,11}$ Impaired mitochondrial function has been linked to physical inactivity, insulin resistance and the pathogenesis of diabetes. ${ }^{10,12}$ Several studies have shown that mitochondrial damage and dysfunction are associated with the development and progression of DKD..$^{713-15}$ Mitochondrial dysfunction leads to alterations in mitochondrial homeostasis, induces membrane depolarization and fragmentation (mitophagy) and bioenergetics dysfunction, producing reactive oxygen species. Hence, Acetyl-CoA Carboxylase (ACC), the intracellular energy stores by the energy sensing kinase AMPK, can be considered to evaluate the bioenergetics process. Lee et al., ${ }^{19}$ showed that a reduction in phosphorylation of ACC is associated with DKD. ${ }^{16-}$ ${ }^{18}$ Tubular injury is associated with reduced mitochondrial function, proposing reduced in the energy, and caused renal fibrosis. ${ }^{4,20}$ Bearing this in mind, in recent years mitochondria have become targets for the development of novel therapies for kidney diseases. However, the mechanisms by which lifestyle interventions, such as exercise, benefit diabetic nephropathy are unknown..$^{21}$

It is known the beneficial of the regular exercise training improvement the cardiovascular, neuroendocrine, respiratory, and musculoskeletal systems. Another hand, the physical inactivity increases the relative risk of several chronic diseases such as coronary artery disease, stroke, diabetes, osteoporosis and some cancers. ${ }^{22}$ Different training modalities have been investigated in diabetes to see if training adaptation is similar in patients and control participants. Importantly, while aerobic exercise training increases mitochondrial biogenesis and oxidative capacity. ${ }^{23-26}$ The benefits of resistance exercise are not limited to enhanced glucose control but also include the maintenance and improvement of muscular strength (force-generating capacity), endurance and power. In addition, increases in lean tissue mass are possible if the resistance exercise induces high muscle forces. ${ }^{26}$ Improved lean tissue mass contributes to the maintenance of basal metabolic rate, promotes functional independence, and potently stimulates increases in skeletal muscle mitochondrial content and mitochondrial biogenesis. ${ }^{27,28}$ Thus, with evidence that physical activity and physical training play important roles in promoting biogenesis and mitochondrial function, and that mitochondria are highly sensitive to signs of contractile activity, our hypothesis is that physical training is able to inhibit evolution of diabetic nephropathy through attenuation and maintenance of mitochondrial quality control through the AMPK / MTF / DRP-1 pathway. 


\section{Research design and methods}

\section{Experimental animals and diabetes induction}

The study protocols were approved by the Ethics in Research Committee at the Federal University of Sao Paulo and all experiments and procedures were in accordance with the National Institutes of Health Guidelines. Male Wistar rats ( 8 weeks old) were divided into eight groups: four non-diabetic groups' non-diabetics (untrainedUN, trained aerobic-TA, trained resistance-TR, trained combined$\mathrm{TC}$ ), diabetic (untrained- UN/D, trained aerobic-TA/D, trained resistance-TR/D, trained combined-TC/D). The animals were housed five per cage with a 12:12-h light-dark cycle, under controlled temperature $\left(26^{\circ} \mathrm{C}\right)$ and had free access to food and water; they were weighed weekly. Diabetes was induced by a single tail injection of Streptozotocin (STZ $50 \mathrm{mg} / \mathrm{kg}$ [iv] - Sigma Chemical Co., St. Louis, $\mathrm{MO}$ ) in freshly prepared $0.01 \mathrm{M}$ citrate buffer, $\mathrm{pH} 4.5$. The animals were not administered insulin or received any treatment apart from exercise training; those with fasting glucose levels of $\geq 250 \mathrm{mg} / \mathrm{dL}$ were included in the study.

\section{Aerobic exercise training protocol}

The aerobic and combined exercise training animals were submitted to an adapted treadmill for 1 week. After this first week, all animals were submitted to a maximum running test to determine the exercise training prescription, and every four weeks to adjust prescription and to evaluate the efficacy of the exercise training protocol. After the test, we designed a training prescription based on 40 to $60 \%$ of the maximal result reached for the effort test. Rats were exercised for $1 \mathrm{~h} /$ day, 5 days a week at a set time each day for 8 weeks.

\section{Resistance exercise training protocol}

The resistance and combined exercise animals underwent training on adapted ladder for one week; 1 section $(6$ climbs with 1 minute of rest between climbs). After this first week, all animals were tested for maximum weight carried to define the exercise training prescription, and every two weeks they were tested to adjust the prescription and evaluate feasibility exercise training protocol. ${ }^{29}$ After the test, we designed a prescription that consisted 40 to $60 \%$ of the maximal $(100 \%)$ obtained produced force.

\section{Combined exercise training protocol}

The groups on to the combined exercise protocol underwent aerobic training on Mondays, Wednesdays and Fridays and resistance training on Tuesdays and Thursdays, following the protocol described above.

\section{Renal parameters - in vivo evaluation}

Two days after the last training session, the rats were housed in individual metabolic cages, and $24 \mathrm{~h}$ urine was collected and centrifuged at 3,000 rpm. To evaluate renal function (proteinuria), a semi-automatic biochemical analyzer (model BIO-200F, São Paulo, Brazil) was used with the Sensiprot Protein Assay Kit (Labtest Diagnóstica SA, Minas Gerais, Brazil).

\section{Protein expression by western blot}

The animals' kidneys were cut into small pieces (30-50mg) and homogenized in RIPA homogenization buffer; Western blotting was performed as described elsewhere. ${ }^{7}$ Membranes were incubated for $2 \mathrm{~h}$ at room temperature with the following primary antibodies: antitotal AMPK, anti-phospho-AMPK, anti-GAPDH, anti-MFN2 and anti-DRP1-1 (Cell Signaling Technology, -Beverly, MA, USA). AntiPGC-1 $\alpha$ antibody was purchased from Santa Cruz Biotechnology (Santa Cruz, CA). Goat anti-rabbit, donkey anti-sheep, donkey antigoat, or goat anti-mouse horseradish peroxidase (HR)-conjugated antibodies were used as secondary antibodies and visualized with an electrochemiluminescence (ECL) detection kit. Values were expressed as fold charge. GAPDH was used as a loading control.

\section{Immunohistochemistry}

Immunohistochemistry was performed using paraffin-embedded kidney sections, which were incubated with anti-ACC antibody overnight at $4^{\circ} \mathrm{C}$. The sections were subsequently incubated with secondary antibodies, treated with diaminobenzidine, counterstained with hematoxylin and examined, as previously reported..$^{30}$

\section{Statistical analysis}

Results of all calculations are expressed as means \pm SE. Data were analyzed using repeated-measures ANOVA with multiple comparisons and Bonferroni's test or one-way ANOVA where appropriate. The results of statistical tests were considered significant if $P$ values were $<0.05$.

\section{Results}

\section{General characteristics of rats}

At the end of the training program, the body weight was reduced in all four diabetic groups when compared with the non-diabetic groups. Unfortunately, the exercise protocol was unable to change these results. However, trained rats' non-diabetics showed a predisposition to a decrease the body weight when compared with untrained Table 1. During the protocol, all the rats given STZ had hyperglycemia than the non- diabetics and higher glucose levels at the end of the protocol than at the beginning Table 1. Irrespective of the type of exercise, after 8 weeks of exercise training the trained animals had an increase in maximum transport weight and/or running distance compared with the untrained animals. In addition, diabetic rats showed reduced maximum weight transported and/or running speed in relation to nondiabetic rats at the end of the protocol Table 2 .

Table I Initial ( 0 week) and final body ( 8 week) weight and glycaemia, kidney weight, urinary protein excretion at the beginning and at the end of the protocol Test $T$ was used to analysis the values $* \mathrm{p}<005$ vs non diabetic (ND) untrained, $\$ \mathrm{p}<005$ vs diabetic (D) untrained final

\begin{tabular}{lllllllll}
\hline & \multicolumn{2}{l}{ Non-trained } & Resistance & \multicolumn{2}{l}{ Aerobic } & & \multicolumn{2}{c}{ Combined } \\
\hline & ND & D & ND & D & ND & D & ND & D \\
\hline Body weight $(\mathrm{g})$ & & & & & & & & \\
Initial & $289 \pm 11$ & $304 \pm 13$ & $325.8 \pm 5$ & $321.7 \pm 6$ & $307.7 \pm 8$ & $298.0 \pm 6$ & $300 \pm 4$ & $310 \pm 6$ \\
Final & $453.8 \pm 16^{*}$ & $294.8 \pm 21^{*}$ & $400.8 \pm 10$ & $276.8 \pm 8^{*}$ & $414.8 \pm 9$ & $281.5 \pm 4^{*}$ & $427.1 \pm 7$ & $297.1 \pm 2 I^{*}$ \\
\hline
\end{tabular}


Table Continued..

\begin{tabular}{|c|c|c|c|c|c|c|c|c|}
\hline & \multicolumn{2}{|c|}{ Non-trained } & \multicolumn{2}{|c|}{ Resistance } & \multicolumn{2}{|l|}{ Aerobic } & \multicolumn{2}{|c|}{ Combined } \\
\hline & ND & D & ND & D & ND & D & ND & D \\
\hline \multicolumn{9}{|l|}{ Glycemic (mg/dL) } \\
\hline Initial & $113 \pm 5$ & $105 \pm 6$ & $107 \pm 3$ & $112 \pm 2$ & $110 \pm 5$ & $10 I \pm 3$ & $100 \pm 3$ & $100 \pm 4$ \\
\hline Final & $104 \pm 3$ & $544 \pm 9 *$ & $\mid I I \pm 2$ & $573 \pm 8 *$ & $107 \pm 2$ & $529 \pm 8 *$ & $101 \pm 2$ & $389 \pm 11 * \$$ \\
\hline Renal weight (g) & $1.38 \pm 0.05$ & $1.84 \pm 0.06^{*}$ & $1.32 \pm 0.04$ & $1.66 \pm 0.05$ & $\mathrm{I} .34 \pm 0.04$ & $1.53 \pm 0.05$ & $1.55 \pm 0.09$ & $1.56 \pm 0.05$ \\
\hline \multicolumn{9}{|c|}{ Proteinuria (g/24hrs) } \\
\hline Initial & $16 \pm 3.6$ & $15 \pm 3.1$ & $15 \pm 1.2$ & $12 \pm 2 . \mid$ & $15 \pm 1.6$ & $17 \pm 1.5$ & $13 \pm 1.4$ & $13 \pm 2.7$ \\
\hline Final & $20 \pm 3.2$ & $52 \pm 4.4 *$ & $15 \pm 1.0$ & $32 \pm 5.1^{\$}$ & $10 \pm 1.7$ & $29 \pm 1.2^{\$}$ & $10 \pm 2.3$ & $34 \pm 3.6^{\$}$ \\
\hline
\end{tabular}

Table 2 Maximum weight and distance tests performed at the beginning and at the end of the protocol, ${ }^{*} p<0.05$ vs. non diabetic (ND) untrained, $\$ p<0.05$ vs. diabetic (D) untrained final

\begin{tabular}{|c|c|c|c|c|c|c|c|c|}
\hline & \multicolumn{2}{|c|}{ Non-trained } & \multicolumn{2}{|l|}{ Resistance } & \multicolumn{2}{|l|}{ Aerobic } & \multicolumn{2}{|c|}{ Combined } \\
\hline & ND & D & ND & D & ND & D & ND & D \\
\hline \multicolumn{9}{|c|}{ Maximal running test(m) } \\
\hline Initial & $154 \pm 19$ & $155 \pm 15$ & NA & NA & $133 \pm 12$ & $136 \pm 20$ & $144 \pm 20$ & $|58 \pm 2|$ \\
\hline Final & $188 \pm 19$ & $50 \pm 3 * \#$ & NA & NA & $332 \pm 12^{* \#}$ & $285 \pm 17 * \#$ & $363 \pm 12^{* \#}$ & $244 \pm 16 * \#$ \\
\hline \multicolumn{9}{|c|}{ Maximal weight test $(g)$} \\
\hline Initial & $369 \pm 15$ & $342 \pm 23$ & $388 \pm 13$ & $383 \pm 20$ & NA & NA & $339 \pm 18$ & $400 \pm 10$ \\
\hline Final & $573 \pm 20^{\#}$ & $4 \mid 4 \pm 26 *$ & $1010 \pm 40^{* \#}$ & $813 \pm 18^{\# \$}$ & NA & NA & $879 \pm 25^{\#}$ & $719 \pm 40^{\# \$}$ \\
\hline
\end{tabular}

\section{Biochemical and morphological features in diabetic renal disease}

After 8 week of the protocol, the kidneys of untrained diabetic rats had significantly higher weights than the kidneys of untrained controls. Exercise specifically lowered kidney weights in trained diabetic rats Table 1 . To evaluate the effects of exercise on renal function abnormalities in diabetic rats, urinary protein excretion rate was used as an index of urinary albumin excretion. Urinary protein excretion was significantly higher in untrained diabetic rats than in their counterparts Table 1. All exercise protocols decreased urinary protein excretion in diabetic rats when compared with untrained peer, but the difference between the trained diabetic groups was not significant. Test $\mathrm{T}$ was used to analysis the values.

\section{Renal mitochondrial}

To investigate changes in mitochondrial fusion and fission, we determined AMPK, PGC1 $\alpha$, Mfn2 and Drp-1 levels in renal mitochondria using Western blot Figures $1 \& 2$. Exercise training can change AMPK expression as it induces glucose uptake and oxidation of fatty acids. This was demonstrated in the present study, the AMPK expression was decreased in untrained diabetic groups, and in the three groups of trained diabetic animals the activity of this enzyme was increased. Figure 1 Based on our finding that mitochondrial biogenesis and induction of PGC-1 $\alpha$ were impaired in the untrained diabetic animals, we can suggest that exercise training may increase expression of this protein in non-diabetic animals and improve PGC$1 \alpha$ expression in diabetic animals, irrespective of the type of exercise Figure 2. Diabetic rats showed impairment in Mfn2 expression that was improved by the exercise training protocol. Furthermore, in the trained control groups, exercise increased Mfn2 expression Figure 2. Drp1 expression was increased, probably leading to stimulation of mitophagy, and exercise training was effective in limiting this stimulation Figure 1. Immunohistochemistry was used to show the activation of ACC in the kidney cortex and medulla. We observed reduced the activation of ACC in the trained diabetic animals when compared with untrained non-diabetic animals, in both in the kidney cortex and medulla Figures 3-5. Surprisingly, the three types of exercise training tested were able to increase ACC activation in the diabetic animals Figures 3-5. 


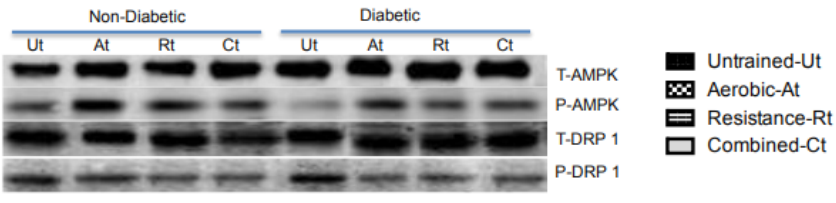

P-AMPK/T-AMPK
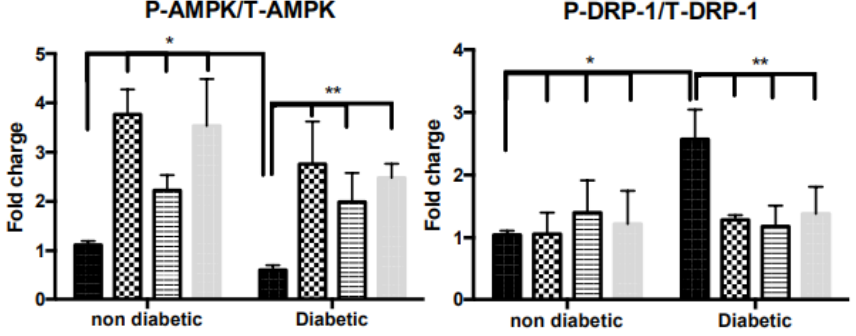

Figure I AMPK and DRP-I protein expression by mitochondrial dynamic pathway were measured in the kidney after exercise training. Data are reported as means \pm SEM. Two way ANOVA, * $\mathrm{P}<0.05$ compared with non diabetic untrained; $\$ \mathrm{P}<0.05$ compared with diabetic untrained.

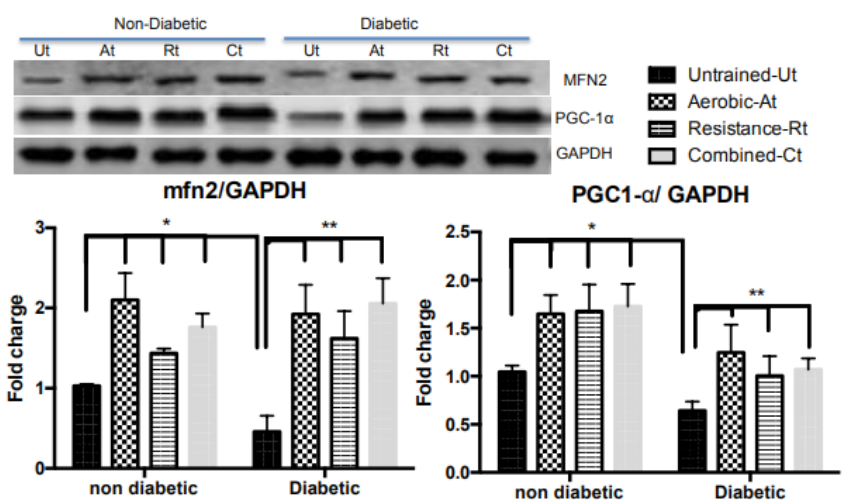

Figure 2 MFN2, PGC-I $\alpha$ and GAPDH protein expression by mitochondrial dynamic pathway was measured in the kidney after exercise training. Data are reported as means \pm SEM. Two- way ANOVA, * $\mathrm{P}<0.05$ compared with non diabetic untrained; $\$ \mathrm{P}<0.05$ compared with diabetic untrained.

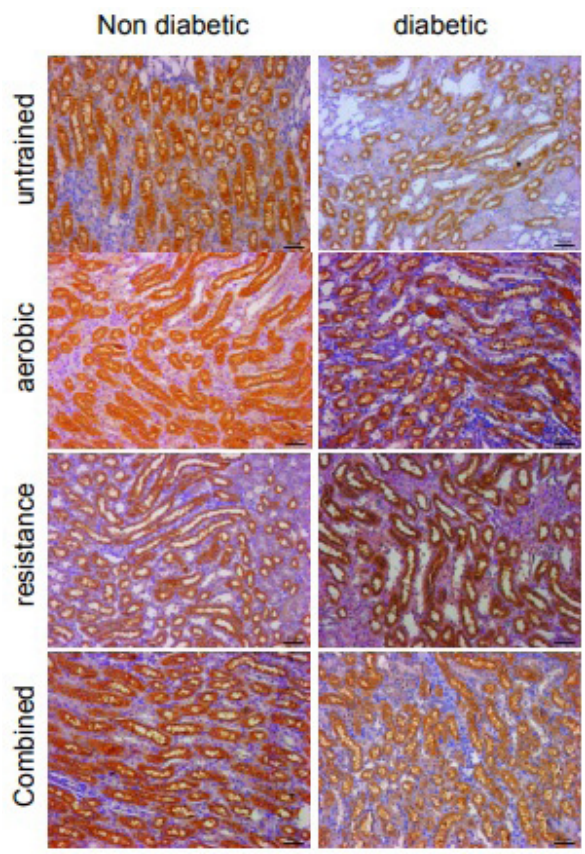

Figure 3 Immunohistochemistry images of the kidney medullar area, using anti-Acetyl-CoA Carboxylase antibodies. Scale bar: $50 \mu \mathrm{m}$.

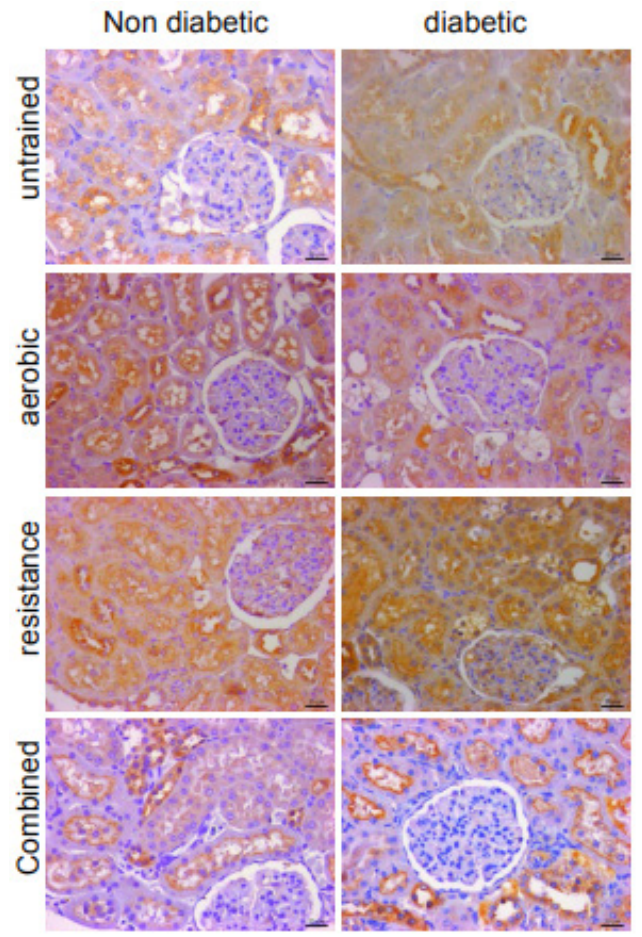

Figure 4 Immunohistochemistry images of the kidney cortex area, using antiAcetyl-CoA Carboxylase antibodies. Scale bar: $25 \mu \mathrm{m}$.
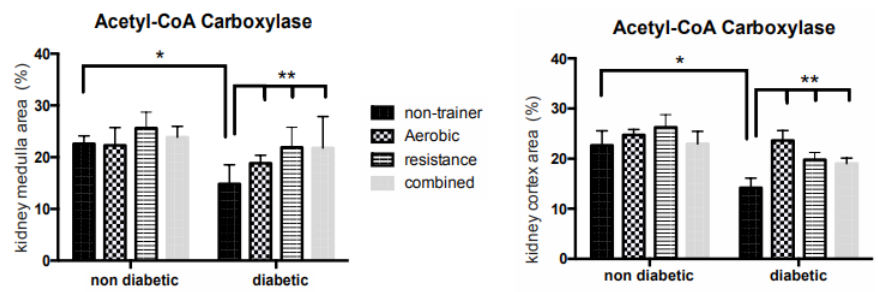

Figure 5 Graphic for Immunohistochemistry of the kidney cortex and kidney medullar area analyzes the acetyl-CoA carboxylase. Data are reported as means \pm SEM. Two-way ANOVA, $*<0.05$ compared with non diabetic untrained; ** $\mathrm{P}<0.05$ compared with diabetic untrained.

\section{Discussion}

The diabetes complications are often responsible for the increase in mortality indices and decrease in quality of life for millions of people worldwide..$^{31}$ Exercise training has been the most prescribed non-pharmacological treatment for the condition. In the present study we showed that the three exercise training types were able to attenuate the severity of diabetic nephropathy. STZ-induced diabetes in rats is characterized by hyperglycemia, excessive osmotic diuresis and weight loss, clinical manifestations similar to those in human diabetes. In addition, STZ-diabetic rats develop chronic microvascular complications, such as nephropathy and peripheral and autonomic neuropathy, which are often also observed in diabetic patients. ${ }^{31,32}$ In the present study, we observed a reduction in body-weight gain in STZ- diabetic rats when they were compared to non-diabetics. However, at the end of the different exercise training programs (aerobic, resistance or combined exercise) the STZ-diabetic rats had maintained their weights, suggesting an improvement in metabolic status. It is important to point out, however, that these animals still had lower body weight than the animals in the non-diabetic groups, and no changes in glucose levels were found between the initial and final values for the same STZ-diabetic group. It is possible that a metabolic compensation system, involving the uptake of proteins and lipids. As 
albuminuria is one of the first clinical features of DN, renal damage was assessed by measuring urinary protein excretion. A significant beneficial influence of exercise training on kidney weight and proteinuria in the exercised diabetic animals was observed, as only one exercise type than combined exercise, was consistently effective in attenuating albuminuria, thus ameliorating renal pathology. This reflects the positive effects of exercise training on $\mathrm{DN}$, as proteinuria is considered one of the main microvascular complications of diabetes. ${ }^{32-34}$ Interestingly exercise induced albuminuria / proteinuria is often reported in normal and diabetic humans. ${ }^{3,35,36}$ This can be attributed to the intensity and duration of exercise, yet exercise training decreases mesangial expansion and glomerular surface area. ${ }^{16}$ However, few studies have been able to provide molecular justifications for this improvement induced by physical training. Several types of exercise can induce considerable changes in metabolism; morphology and mitochondrial function in muscle and heart. ${ }^{37} \mathrm{~A}$ wide variety of exercise interventions have been reported in the management of diabetes and have shown beneficial metabolic and mitochondrial changes. Nevertheless, it is still unknown how exercise influences the mitochondria in the kidney.

While our results showed an increase in running time and strength in the exercise trained diabetic animals, the untrained diabetes animals showed lower scores in these tests at the end of the training program than at the beginning, indicating loss of exercise capacity after diabetes induction. Resistance trained diabetic animals showed increased strength capacity at the end of the protocol. This demonstrates that resistance training was effective, at least in promoting the maintenance of functional capacity in these animals. All animals had strength gains during the ladder exercise protocol, probably because of muscle growth during that week and, consequently, favorable changes in muscle properties, resulting in increased capacity to generate force. ${ }^{38}$ These gains were attenuated in the animals of the untrained groups, probably because of the loss in skeletal muscle mass in STZ-induced diabetes. ${ }^{39}$ The animals in the resistance training and combined training groups were able to climb the ladder with loads higher than the animals in the other groups and showed an increase in the mass of the plantaris muscle, indicating the effectiveness of the protocol based on the maximum load test. It is important to note that although diabetic patients generally present with reduced strength and aerobic capacity, ${ }^{40,41}$ after combined exercise training (performed on alternate days on treadmill and ladder) animals show greater ability to run and reach the maximum load in the ladder climbing. Mitochondrial content is determined by a balance between mitochondrial biogenesis (synthesis) and degradation (mitophagy). ${ }^{42}$ It is recognized that the mitochondria of patients with type 2 diabetes exhibit morphological and biochemical signs suggestive of mitochondrial dysfunction. ${ }^{43-45}$

It has been shown that chronic exercise induces an increase in mitochondrial volume and function, improving workability and fatigue resistance in various organs. ${ }^{46,47}$ This adaptability of the organelle allows us to be optimistic about the role of exercise in the prevention of mitochondrial dysfunction. Understanding the underlying mechanisms of diabetes-related mitochondrial dysfunction led to the study of parallel preventive and therapeutic strategies based on non-pharmacological approaches. Our results complement this data by demonstrating that periodic exercise influences the cellular mechanisms involved in the increase of mitochondrial volume. PGC$1 \alpha$ is involved in the regulation of thermogenesis, energy metabolism and other biological processes that are critical for the control of the phenotypic characteristics of various organs. The most prominent strategy to prevent and reverse some of the mitochondrial defects of the diabetic phenotype is the over-regulation of this protein, ${ }^{48,49}$ since it is normally negatively regulated in diabetic patients and is one of the probable causes of the oxidative capacity of these patients. ${ }^{6}$ The regulation of PGC-1 $\alpha$ may be potentially useful in the prevention and treatment of diabetes..$^{50}$ Indeed, our results showed that diabetes decreased PGC- $1 \alpha$, as previously described, and physical training was effective to positively regulate this protein in the non-diabetic groups, as well as attenuate the impairment in energy metabolism in the diabetic rats. One of the most important mechanisms related to PGC- $1 \alpha$ gene expression is induced by increased activation (i.e., phosphorylation) of AMPK-sensitive. AMPK, a key sensor of metabolic stress, has been identified as a potential target for the treatment of insulin resistance and type 2 diabetes. ${ }^{49}$ As stimulation of the AMPK pathway leads to increased biogenesis and mitochondrial function, ${ }^{18}$ strategies to achieve this goal may include exercise and stimuli of adenosine monophosphate kinase activation. ${ }^{51,52}$ However, AMPK activation is more pronounced during / immediately after exercise. We found a significant increase in the expression of this protein in all trained animals, showing a reversal of the damage caused by diabetes.

Maintaining mitochondrial shape, size, network and function requires autophagic degradation and the removal of damaged mitochondria through the process of mitophagy, which is active at baseline, during development and under stress conditions. ${ }^{15,53}$ Among the proteins involved in mitochondrial dynamics, the most cited are two mitochondrial guanine triphosphatases (GTPases) related to fusion and fission: $\mathrm{Mfn} 2^{10}$ and Drp $1,{ }^{54}$ respectively. Endurance exercise increases Mfn2 $\mathrm{mRNA}^{51}$ and phosphorylation of Drp ${ }^{43}$ in human skeletal muscle. To the authors' knowledge, no other reports of the action of these proteins in the kidney of exercised diabetic animals have been published. We found that physical training increased the expression of Mfn2 in the non-diabetic groups and prevented the reduction in this protein expression caused by diabetes. In addition, we showed that exercise reduces the phosphorylation of Drp1, although it was still positively regulated in the groups of diabetic animals trained, this difference was higher and statistically significant between the untrained diabetic group. Mitochondria play a vital role in maintaining health through their regulation of substrate metabolism and energy production. It is highly sensitive to contraction- initiated signals, physical activity and exercise play important roles in promoting biogenesis and mitochondrial function helping to maintain cellular and body health. The main mitochondrial function is fatty acid oxidation, which is the main source of energy. ACC is an important regulator of fatty acid synthesis and oxidation rates and it is involved in energy homeostasis. ${ }^{55}$ Currently, two isoforms, ACC1 and ACC2, are known. While ACC2 is more expressed in the heart, muscles and liver and is associated with the mitochondrial membrane, cytoplasmic ACC1 is expressed mainly in the kidney. ${ }^{19}$ The reduction in ACC1 has been associated with renal injury. It has also been shown that the activation of ACC by AMPK reduces renal damage. ${ }^{19}$

Our study demonstrated that the chronic effect of aerobic exercise, resistance exercise and combined exercise training positively influences renal ACC. While diabetes reduces ACC activation, as already shown in the literature, the 8 weeks of exercise were able to increase the activation of this protein. More importantly, we observed that the responses to exercise were similar in the cortex and renal medulla. In conclusion, the results of this study show that aerobic training, resistance training or combined training lead to a reduction in complications in induced diabetes in rats, probably as a result of increased physical capacity and vascular and autonomic benefits. The combined training protocol promoted more benefits than the aerobic and resistance protocols separately. We emphasize that all the 
improvements observed were independent of glycemic index control. Our findings allow us to infer that the mechanism behind the protective effects of physical training on renal function observed involves the regulation of mitochondrial dynamics and the improvement in the activation of ACC by AMPK. The clinical application of exercise training in early diabetic patients may help to prevent and / or treat diabetic nephropathy. ${ }^{56}$

\section{Acknowledgments}

None.

\section{Conflicts of interest}

The authors declare that they have no conflicts of interest.

\section{Funding}

This study was supported by Fundacao de Amparo a Pesquisa do Estado de Sao Paulo (FAPESP) and Conselho Nacional de Desenvolvimento Cientıfico e Tecnologico (CNPq).

\section{References}

1. Eirin A, Lerman A, Lerman LO. The emerging role of mitochondria targeting in kidney disease. Handb Exp Pharmacol. 2017;240:229-250.

2. Larsen S, Skaaby S, Helge JW, et al. Effects of exercise training on mitochondrial function in patients with type 2 diabetes. World J Diabetes. 2014;5(4):482-492.

3. Simpson K, Wonnacott A, Fraser DJ, et al. MicroRNAs in diabetic nephropathy: from biomarkers to therapy. Curr Diab Rep. 2016;16(3):35.

4. Watanabe J, Takiyama Y, Honjyo J, et al. Role of IGFBP7 in diabetic nephropathy: TGF-beta1 induces IGFBP7 via Smad2/4 in human renal proximal tubular epithelial cells. PloS One. 2016;11(3):e0150897.

5. Kelley DE, He J, Menshikova EV, et al. Dysfunction of mitochondria in human skeletal muscle in type 2 diabetes. Diabetes. 2002;51(10):2944 2950

6. Richter EA, Ruderman NB. AMPK and the biochemistry of exercise: implications for human health and disease. Biochem J. 2009;418(2):261275 .

7. Tang WX, Wu WH, Qiu HY, et al. Amelioration of rhabdomyolysisinduced renal mitochondrial injury and apoptosis through suppression of Drp-1 translocation. J Nephrol. 2013;26(6):1073-1082.

8. Tang WX, Wu WH, Zeng XX, et al. Early protective effect of mitofusion 2 overexpression in STZ-induced diabetic rat kidney. Endocrine. 2012;41(2):236-247.

9. Winder WW, Hardie DG. AMP-activated protein kinase, a metabolic master switch: possible roles in type 2 diabetes. Am J Physiol. 1999;277(1):E1-E10

10. Finck BN, Kelly DP. PGC-1 coactivators: inducible regulators of energy metabolism in health and disease. J Clin Invest. 2006;116(3):615-622.

11. Liang H, Ward WF. PGC-1alpha: a key regulator of energy metabolism. Adv Physiol Educ. 2006;30(4):145-151.

12. Handschin C, Spiegelman BM. The role of exercise and PGC1alpha in inflammation and chronic disease. Nature. 2008;454:463-469.

13. Munusamy S, MacMillan-Crow LA. Mitochondrial superoxide plays a crucial role in the development of mitochondrial dysfunction during high glucose exposure in rat renal proximal tubular cells. Free Radic Biol Med. 2009;46(8):1149-1157

14. Sun L, Xie P, Wada J, et al. Rap1b GTPase ameliorates glucose-induced mitochondrial dysfunction. J Am Soc Nephrol. 2008;19(12):2293-2301.
15. Twig G, Shirihai OS. The interplay between mitochondrial dynamics and mitophagy. Antioxid Redox Signal. 1939;14(10):1939-1951.

16. Coughlan MT, Nguyen TV, Penfold SA, et al. Mapping time-course mitochondrial adaptations in the kidney in experimental diabetes. $\mathrm{Clin} \mathrm{Scl}$ (Lond). 2016;130(9):711-720.

17. Hou Y, Li S, Wu M, et al. Mitochondria-targeted peptide SS-31 attenuates renal injury via an antioxidant effect in diabetic nephropathy. Am J Physiol Renal Physiol. 2016;310(6):F547-F559.

18. Li L, Mühlfeld C, Niemann B, et al. Mitochondrial biogenesis and PGC$1 \alpha$ deacetylation by chronic treadmill exercise: differential response in cardiac and skeletal muscle. Basic Res Cardiol. 2011;106(6):1221-1234.

19. Lee M, Katerelos M, Gleich K, et al. Phosphorylation of acetyl-CoA carboxylase by AMPK reduces renal fibrosis and is essential for the antifibrotic effect of metformin. J Am Soc Nephrol. 2018;29(9):2326-2336.

20. Higgins GC, Coughlan MT. Mitochondrial dysfunction and mitophagy: the beginning and end to diabetic nephropathy? $\mathrm{Br} J$ Pharmacol. 2014;171(8):1917-1942.

21. Ghosh S, Khazaei M, Moien-Afshari F, et al. Moderate exercise attenuates caspase-3 activity, oxidative stress, and inhibits progression of diabetic renal disease in db/db mice. Am J Physiol Renal Physiol. 2009;296(4):F700-F708.

22. Katzmarzyk PT, Janssen I. The economic costs associated with physical inactivity and obesity in Canada: an update. Can $J \mathrm{Appl}$ Physiol. 2004;29(1):90-115.

23. Constable SH, Favier RJ, McLane JA, et al. Energy metabolism in contracting rat skeletal muscle: adaptation to exercise training. $\mathrm{Am} J$ Physiol. 1987;253(2 Pt 1):C316-C322.

24. Gollnick PD, Armstrong RB, Saltin B, et al. Effect of training on enzyme activity and fiber composition of human skeletal muscle. J Appl Physiol. 1972;34(1):107-111.

25. Holloszy JO. Biochemical adaptations in muscle. Effects of exercise on mitochondrial oxygen uptake and respiratory enzyme activity in skeletal muscle. J Biol Chem. 1967;242(9):2278-2282.

26. LaStayo PC, Woolf JM, Lewek MD, et al. Eccentric muscle contractions: their contribution to injury, prevention, rehabilitation, and sport. J Orthop Sports Phys Ther. 2003;33(10):557-571.

27. Haight T, Tager I, Sternfeld B, et al. Effects of body composition and leisure-time physical activity on transitions in physical functioning in the elderly. Am J Epidemiol. 2005;162(7):607-617.

28. Pratley R, Nicklas B, Rubin M, et al. Strength training increases resting metabolic rate and norepinephrine levels in healthy 50- to 65-yr-old men. J Appl Physiol. 1994;76(1):133-137.

29. Silva KAS, Arlotti MR, Jorge L, et al. Progressive resistance exercise training attenuated renal damages, but did not improve muscle force in STZ-Induced diabetic rats. J Diabetes Metab. 2014;5(11):1000461.

30. Kang KP, Kim DH, Jung YJ, et al. Alpha-lipoic acid attenuates cisplatininduced acute kidney injury in mice by suppressing renal inflammation. Nephrol Dial Transplant. 2009;24(10):3012-3020.

31. Defronzo RA. Banting lecture. From the triumvirate to the ominous octet: a new paradigm for the treatment of type 2 diabetes mellitus. Diabetes. 2009;58(4):773-795.

32. Harthmann AD, De Angelis K, Costa LP, et al. Exercise training improves arterial baro- and chemoreflex in control and diabetic rats. Auton Neurosci. 2007;133(2):115-120.

33. Akbarzadeh A, Norouzian D, Mehrabi MR, et al. Induction of diabetes by streptozotocin in rats. Indian J Clin Biochem. 2007;22(2):60-64.

34. Schmidt RE, Plurad SB. Ultrastructural and biochemical characterization of autonomic neuropathy in rats with chronic streptozotocin diabetes. $J$ Neuropathol Exp Neurol. 1986;45:525-544. 
35. Kohzuki M, Kamimoto M, Wu XM, et al. Renal protective effects of chronic exercise and antihypertensive therapy in hypertensive rats with chronic renal failure. J Hypertens. 2001;19(10):1877-1882.

36. Melendez-Ramirez LY, Richards RJ, Cefalu WT. Complications of type 1 diabetes. Endocrinol Metab Clin North Am. 2010;39(3):625-640.

37. Heathcote KL, Wilson MP, Quest DW, et al. Prevalence and duration of exercise induced albuminuria in healthy people. Clin Invest Med. 2009;32(4):E261-E265.

38. Koh KH, Dayanath B, Doery JC, et al. Effect of exercise on albuminuria in people with diabetes. Nephrology (Carlton). 2011;16(8):704-709.

39. Henriksen EJ. Invited review: effects of acute exercise and exercise training on insulin resistance. J Appl Physiol. 2002;93(2):788-796.

40. Roberts CK, Won D, Pruthi S, et al. Effect of a diet and exercise intervention on oxidative stress, inflammation and monocyte adhesion in diabetic men. Diabetes Res Clin Pract. 2006;73(3):249-259.

41. Short KR, Vittone JL, Bigelow ML, et al. Impact of aerobic exercise training on age-related changes in insulin sensitivity and muscle oxidative capacity. Diabetes. 2003;52(8):1888-1896.

42. Melser S, Lavie J, Bénard G. Mitochondrial degradation and energy metabolism. Biochim Biophys Acta. 2015;1853(10 Pt B):2812-2821.

43. Kramerova I, Kudryashova E, Wu B, Germain et al. Mitochondrial abnormalities, energy deficit and oxidative stress are features of calpain 3 deficiency in skeletal muscle. Hum Mol Genet. 2009;18(17):3194-3205.

44. Xiao L, Xu X, Zhang F, et al. The mitochondria-targeted antioxidant MitoQ ameliorated tubular injury mediated by mitophagy in diabetic kidney disease via Nrf2/PINK1. Redox Biol. 2017;11:297-311.

45. Yu T, Sheu SS, Robotham JL, et al. Mitochondrial fission mediates high glucose- induced cell death through elevated production of reactive oxygen species. Cardiovascu Res. 2008;79(2):341-351.

46. Yan Z, Lira VA, Greene NP. Exercise training-induced regulation of mitochondrial quality. Exerc Sport Sci Rev. 2012;40(3):159-164.
47. Yan Z, Okutsu M, Akhtar YN, et al. Regulation of exercise-induced fiber type transformation, mitochondrial biogenesis, and angiogenesis in skeletal muscle. J Appl Physiol. 2011;110(1):264-274.

48. Adhihetty PJ, Uguccioni G, Leick L, et al. The role of PGC-1alpha on mitochondrial function and apoptotic susceptibility in muscle. $\mathrm{Am} \mathrm{J}$ Physiol Cell Physiol. 2009;297(1):C217-C225.

49. Benton CR, Holloway GP, Han XX, et al. Increased levels of peroxisome proliferator-activated receptor gamma, coactivator 1 alpha (PGC1alpha) improve lipid utilisation, insulin signalling and glucose transport in skeletal muscle of lean and insulin-resistant obese Zucker rats. Diabetologia. 2010;53(9):2008-2019.

50. Audzeyenka I, Rachubik P, Typiak M, et al. Hyperglycemia alters mitochondrial respiration efficiency and mitophagy in human podocytes. Exp Cell Res. 2021;407(1):112758.

51. Cartoni R, Leger B, Hock MB, et al. Mitofusins $1 / 2$ and ERRalpha expression are increased in human skeletal muscle after physical exercise. J Physiol. 2005;567(Pt 1):349-358.

52. Siciliano G, Simoncini C, Lo Gerfo A, et al. Effects of aerobic training on exercise-related oxidative stress in mitochondrial myopathies. Neuromuscul Disord. 2012;22 Suppl 3(3-3):S172-S177.

53. Youle RJ, Narendra DP. Mechanisms of mitophagy. Nat Rev Mol Cell Biol. 2011;12(1):9-14.

54. Sumida M, Doi K, Ogasawara E, et al. Regulation of mitochondrial dynamics by dynamin-related protein- 1 in acute cardiorenal syndrome. $J$ Am Soc Nephrol. 2015;26(10):2378-2387.

55. Chen L, Duan Y, Wei H, et al. Acetyl-CoA carboxylase (ACC) as a therapeutic target for metabolic syndrome and recent developments in ACC1/2 inhibitors. Expert Opin Investig Drugs. 2019;28(10):917-930.

56. de Brito OM, Scorrano L. Mitofusin 2: a mitochondria-shaping protein with signaling roles beyond fusion. Antioxid Redox Signal. 2008;10(3):621-633. 